\title{
The Reproducibility of a Kinematically-Derived Axis of the Knee versus Digitized Anatomical Landmarks using a Knee Navigation System
}

\author{
Lisa Case Doro ${ }^{1}$, Richard E. Hughes, ${ }^{*,}$, Joshua D. Miller ${ }^{1}$, Karl F. Schultz ${ }^{1}$, Brian Hallstrom ${ }^{2}$, \\ Andrew G. Urquhart ${ }^{1}$
}

${ }^{I}$ Department of Orthopaedic Surgery, University of Michigan, Ann Arbor, MI, USA

${ }^{2}$ St. Joseph Mercy Health Center, Orthopedic Surgery Associates, P.C., Ann Arbor, MI, USA

\begin{abstract}
Component position is critical to longevity of knee arthroplasties. Femoral component rotation is typically referenced from the transepicondylar axis (TEA), the anterior-posterior (AP) axis or the posterior condylar axis. Other studies have shown high variability in locating the TEA while proposing digitization of other landmarks such as the AP axis as a less-variable reference. This study uses a navigation system to compare the reproducibility of computing a kinematically-derived, navigated knee axis (NKA) to digitizing the TEA and AP axis. Twelve knees from unembalmed cadavers were tested. Four arthroplasty surgeons digitized the femoral epicondyles and the AP axis direction as well as flexed and extended the knee repeatedly to allow for NKA determination. The variance of the NKA axis determined under neutral loading conditions was smaller than the variance of the TEA axis when the kinematics were measured in the closed surgical condition $(\mathrm{P}<0.001)$. However, varus, valgus, and internal loading of the leg increased the variability of the NKA. Distraction of the leg during knee flexion and extension preserved the low variability of the NKA. In conclusion, a kinematically-derived NKA under neutral or distraction loading is more reproducible than the TEA and AP axis determined by digitization.
\end{abstract}

Keywords: Knee navigation system, knee arthroplasty, component position.

\section{INTRODUCTION}

Correct component position is critical to the performance and longevity of total knee arthroplasties (TKA) [1,2]. For example, setting the femoral component rotation parallel to the transepicondylar axis (TEA) optimizes patello-femoral and tibio-femoral articulations [3,4]. This has traditionally been achieved by intra-operatively assessing the femoral epicondyles and/or the femoral anteroposterior (AP) axis (often called Whiteside's Line) and using these landmarks to determine the TEA. Many studies have demonstrated that computer-assisted knee replacement results in more accurate positioning of components than traditional jig-based techniques [5-9]. Despite the relative improvement, computerassisted systems used for surgical navigation during a total knee replacement rely on intra-operative palpation and digitization of landmarks about the knee. For identifying the TEA, this method may not be optimally repeatable. Jenny and Boeri, using two surgeons and a passive-marker navigation system, found that digitization of the TEA yielded mean intra-observer ranges of variation of $5^{\circ}$ and $6^{\circ}$ for the two surgeons along with a mean inter-observer range of variation of $9^{\circ}$ [10]. Katz and Beck et al. found the axis $90^{\circ}$ to the digitized femoral AP axis to be more accurate and reproducible than the TEA found by palpating and digitizing the femoral epicondyles in optimally setting the rotational alignment of the femoral component in TKA [11].

*Address correspondence to this author at the Laboratory for Optimization and Computation in Orthopaedic Surgery, Department of Orthopaedic Surgery, University of Michigan, 2019 BSRB, 109 Zina Pitcher Pl., Ann Arbor, MI 48109-2200, USA; E-mail: rehughes@umich.edu
The use of a navigation system makes it possible to derive a different reference, a kinematically-determined reference axis, which we will term the navigated knee axis or NKA, intra-operatively. The purpose of the study was to test the hypothesis that the navigated knee axis is a more reproducible reference axis than the manually digitized TEA or AP axis. We focused on the repeatability of axis determination rather than accuracy because we believe reducing outliers in component alignment is a critical strategy for improving TKA longevity. If it is in fact more reproducible than other standard reference landmarks, then subsequent studies could be performed to determine how the femoral bone cuts should be made relative to the NKA. While our study does not provide a complete statement of how the cuts should be made, it does provide evidence that a method using the NKA would be based on a reproducible axis. Ideally, the NKA would not be affected by inadvertent loading of the leg by the surgeon. Thus, we also sought to determine the effects of leg loading on NKA variability. The effect of ACL deficiency and arthrotomy was also briefly investigated.

\section{MATERIALS AND METHODOLOGY}

A cadaver study was conducted in which three of four arthroplasty surgeons were blinded to the purpose of the study. Twelve knees of twelve fresh, frozen, whole human cadavers ( 5 males, 7 females; mean age 79.2, range 47-95 y/o) were tested. Exclusion criteria were a flexion contracture of more than $10^{\circ}$ or prior knee surgery. All but two, which were normal, had varying degrees of osteoarthritis from mild to severe. Nine of the twelve had moderate to severe disease that would have warranted TKA. Upon ar- 
throtomy, it was noted that the arthritic knees had osteophytes, some were ACL deficient and/or had degenerative medial or lateral meniscus tears. When both knees fit our criteria, a side was chosen with a coin toss.

Data were collected using the Stryker knee navigation system (Stryker Corporation, Kalamazoo, MI). This navigation system is an active, infrared, motion capture system that uses digitized anatomical landmark data to construct a model of the knee and calculate where and at what angle bone cuts should be located. To allow the system to localize each bone in 3D space, two infrared trackers were affixed to the cadaver limb with bicortical screws, one anteriorly on the femur and the other anteriorly on the tibia. The system was modified with custom research software to allow the capture of additional data. Thus, in addition to collecting the survey of anatomical landmarks typically performed clinically, the system was also customized to record full ranges of knee motion as well as additional landmark digitization (i.e. multiple collections of epicondyle locations and AP axis directions). Collection of the range of motion data allowed for the subsequent calculation of the navigated knee axis. The specimen's foot was wrapped in a boot which had a metal eyelet attached to the bottom of it. A range-of-motion trial was defined as a single surgeon manually cycling the knee through three full ranges of motion, from full extension to flexion beyond $90^{\circ}$ back to extension. For each trial, a navigated knee axis was computed.

To test how robust the kinematic method was to loading and surgical condition, the surgeons repeated the three full ranges of motion for each applied loading and surgical condition. There were three surgical conditions: closed (no incisions), ACL (a small medial arthrotomy was made leaving the medial collateral ligament origin and meniscus intact, and the ACL was divided, if present), and open (a standard medial para-patellar incision was made to expose the knee joint). To keep the surgical conditions consistent from one specimen to the next, the senior author always performed the exposures and ACL division and then carried out his navigation trials first. The three additional surgeons, who were all experienced in total knee replacement and blinded to the purpose of the study, were randomly ordered in performing the navigation trials for each specimen.

For each surgical condition, the surgeons were asked to bring the knee through the range-of-motion trials, each with a different applied load: neutral (no applied load), valgus, varus, internal rotation, external rotation, and a distraction force (Fig. 1). The valgus, varus, internal and external rotation loads were applied manually by having the surgeon place one hand on the distal femur and the other on the distal tibia and either bending or twisting the leg in the appropriate direction for the requested load throughout the range of motion. The distraction force was a tensile load of greater than $45 \mathrm{~N}$ applied by hooking a spring scale to the eyelet at the bottom of the boot and pulling throughout the range of motion. To evaluate the variability of determining the navigated knee axis, each surgeon performed three trials of each applied load for the closed and ACL surgical conditions. For the open condition, only the senior author repeated each applied load three times while the remaining surgeons performed only one trial per applied load. This was done to cut time out of the protocol, and variability in the open status was not deemed as important because the navigated knee axis could be determined prior to the arthrotomy.

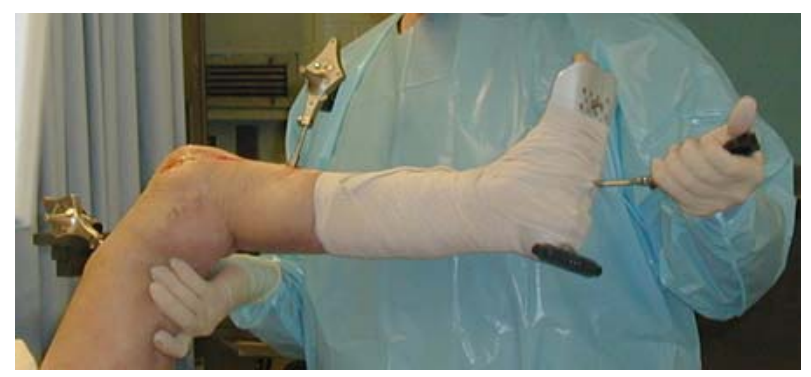

Fig. (1). Applying distraction force using a spring scale during kinematic determination of the navigated knee axis.

After the range-of-motion trials and once the knee was fully exposed, each surgeon was asked to palpate and then digitize the sulcus of the medial epicondyle and prominence of the lateral epicondyle with the digitizing tool (Fig. 2). To understand how variable the digitization of the TEA was, they were asked to do this three times each, nonconsecutively. Each surgeon was also asked to point the digitizer tip along the direction of the AP axis twice, nonconsecutively. Additionally, the hip center was calculated by the navigation system while the surgeon rotated the femoral head in the acetabulum. This point was used along with the digitized knee center to define the mechanical axis of the femur.

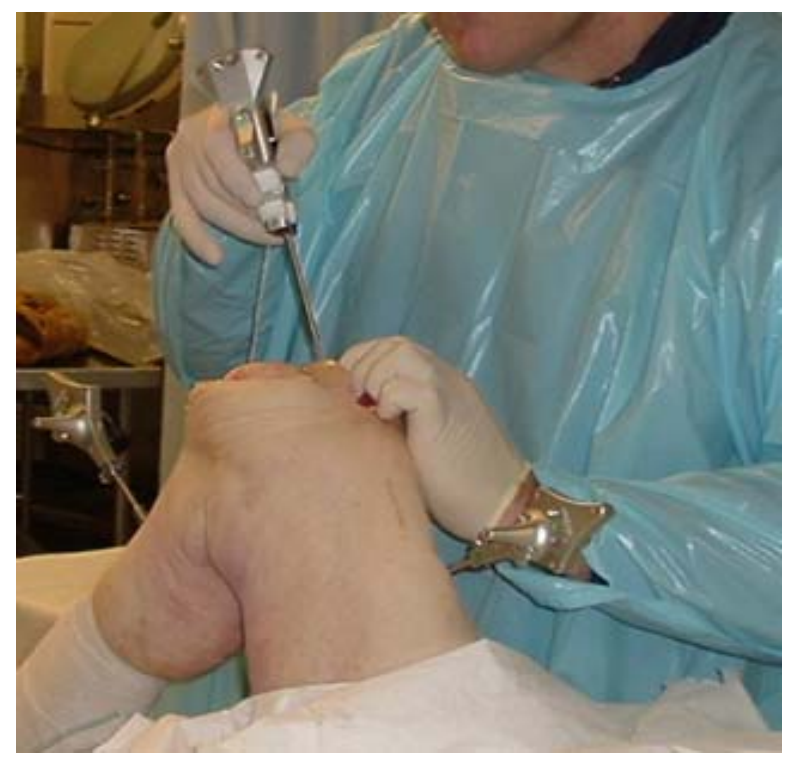

Fig. (2). Surgeon digitizing lateral epicondyle.

Instructions and graphics from the Stryker Navigation manual were photocopied and enlarged for the surgeons to follow when digitizing landmarks; no additional guidance was provided. Specifically for the epicondyles, the instructions were as follows: "Point digitizer tip to the prominence of the lateral epicondyle" and "point digitizer tip to the sulcus of the medial epicondyle." For the femoral AP axis, the instructions were to "align the digitizer tip with the patient's sulcus of the infratrochlear groove." The surgeons were kept 
in a separate room when they were not actively ranging the knee or digitizing landmarks so they were unable to observe one another.

\section{Data Analysis}

Stryker navigation software processed the flexionextension kinematic data to generate a reference axis, the navigated knee axis, for each range of motion trial (three cycles of flexion and extension by a given surgeon for a given surgical and loading state). This axis is the average instantaneous helical axis through the arc of motion. It is computed by smoothing the kinematic data using cubic splines, forming a local femoral coordinate system, transforming data into the local coordinate system, subdividing the arc of motion into three-degree increments, computing the helical axis for each interval, and averaging the axis over the intervals. Helical axis computations for each interval are performed using the method proposed by Spoor and Veldpaus [12].

Because this navigated knee axis was generated with respect to a specimen-specific coordinate system, a relative location to a reference line, which is described below, was deemed useful in comparing specimen to specimen.

The TEA is a vector with direction and magnitude. Since it is used in this setting to determine rotation of the femoral component around the mechanical axis, only the projection of the TEA onto the plane perpendicular to the mechanical axis is relevant for this study.

The mechanical axis was determined by digitizing the knee center and kinematically calculating the hip center. It is possible to define the position of the TEA in this plane only with respect to a "reference line." In theory, any reference line could be used. For ease of analysis and to facilitate comparison between specimens, in this study the reference line will be defined as the average of all digitized TEA measurements for that specimen. The positions (in the plane perpendicular to the mechanical axis) of the individual navigated knee axes and of the individual digitized TEAs were subtracted from the position of this reference line to generate an "angle difference" (Fig. 3). A positive angle difference indicates the navigated knee axis is externally rotated compared to the average TEA. A negative angle difference would indicate internal rotation compared to the average TEA.

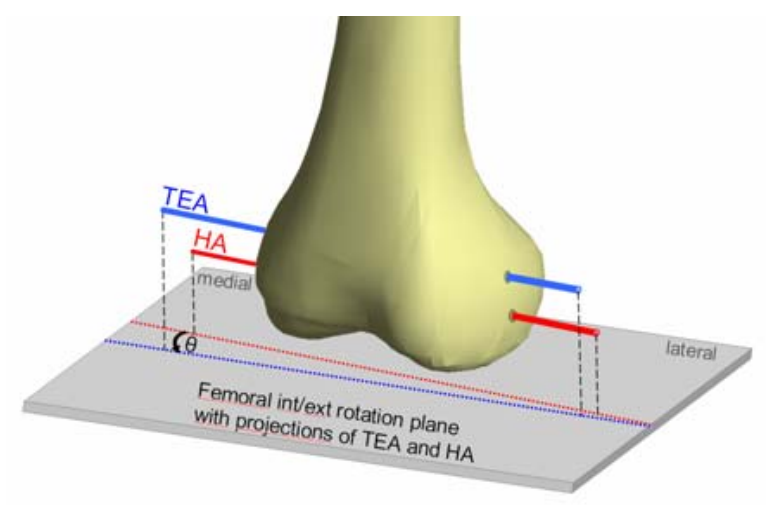

Fig. (3). Antero-lateral view of the left distal femur. $\theta$ is the angle between the projections of the transepicondylar axis (TEA) and the navigated knee axis (NKA) onto the axial plane.
Inter- and intra-surgeon variability was evaluated for the kinematically-derived navigated knee axes as well as the digitized TEA and digitized AP axis.

\section{Statistical Methods}

F-tests were used to test for equality of variances. A statistical significance level of 0.05 was used, but the reader can perform their own assessment of the overall Type I error by noting that a total of seven F-tests were performed. Intraclass correlation coefficients (ICCs) were also computed. Data manipulation was performed in MATLAB (The Mathworks Inc., Natick, MA) and statistical analyses were performed using MINITAB (MINITAB Inc., State College, PA).

\section{RESULTS}

The variance of the NKA axis determined under neutral loading conditions was smaller than the variance of the TEA axis when the kinematics were measured in the closed surgical condition $(\mathrm{P}<0.001)$. The 95\% confidence intervals for the standard deviation of the TEA and NKA under neutral loading were [3.05, 3.85] and [2.14, 2.71], respectively (Fig. 4).

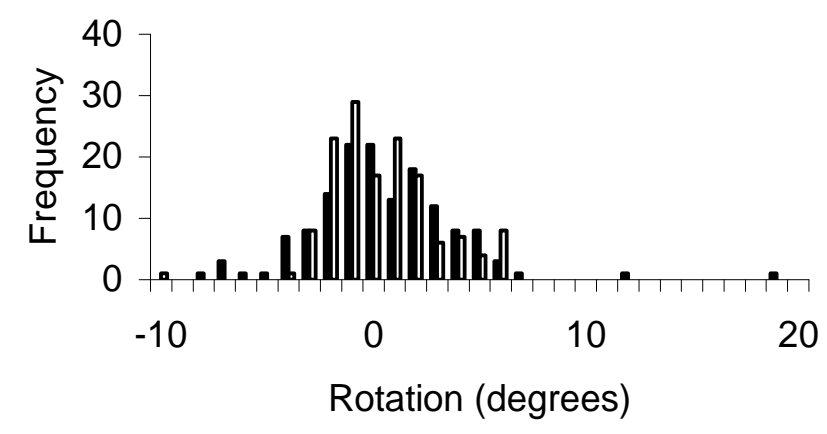

Fig. (4). Spread of TEA axis (solid bars) and NKA axis measured under neutral loading condition (hollow bars), represented as histograms. Note TEA outliers.

Compared to the variance of the NKA in the neutral loading condition, the variance of the NKA was larger for varus loading $(\mathrm{P}<0.001)$, valgus loading $(\mathrm{P}<0.004)$, and internal rotation loading $(\mathrm{P}<0.001)$ (Table 1). However, no difference in variance was found between the NKA under neutral loading and the NKA computed from trials involving external rotation loading $(\mathrm{P}=0.122)$ or axial loading using a distraction force $(\mathrm{P}=0.288)$. There is significant overlap of the confidence intervals of the standard deviation for the distraction $([1.96,2.47])$ and neutral loading $([2.14,2.71])$ conditions. The variance of the AP axis was also larger than the NKA measured under neutral loading $(\mathrm{P}<0.001)$ : the standard deviation had a $95 \%$ confidence interval of [3.93, 5.24]. The average difference between the NKA and TEA axes was 0.04 degrees of internal rotation, which means the NKA was very slightly internally rotated compared to the average TEA. The standard deviation of that measure across subjects was 2.03 degrees. The inter- and intra-surgeon reliability was better for the NKA under normal loading than the TEA. The inter-surgeon reliability, as measured by the intraclass correlation coefficient (ICC), was 0.43 and 0.60 for the TEA and NKA, respectively. The intra-surgeon ICC's were -0.13 and 0.84 for the TEA and NKA, respectively. The $95 \%$ confi- 
dence intervals of the NKA standard deviation measured during the closed, ACL transected, and arthrotomy surgical states were $[2.14,2.71],[2.15,4.62]$, and $[2.09,2.91]$, respectively.

Table 1. 95\% Confidence Intervals for the Standard Deviation of the NKA axis (Degrees)

\begin{tabular}{|c|c|c|}
\hline Loading & Std. Dev. & 95\% C.I. \\
\hline \hline neutral & 2.39 & {$[2.14,2.71]$} \\
\hline valgus & 3.05 & {$[2.74,3.45]$} \\
\hline varus & 4.13 & {$[3.70,4.67]$} \\
\hline internal rotation & 5.70 & {$[5.10,6.44]$} \\
\hline external rotation & 2.10 & {$[1.88,2.38]$} \\
\hline distraction & 2.19 & {$[1.96,2.47]$} \\
\hline
\end{tabular}

\section{DISCUSSION}

An important aspect of component positioning in total knee arthroplasty is setting femoral component rotation. Navigation systems using digitization of the femoral epicondyles to determine the TEA are based on the ability to locate the epicondyles, but Jenny and Boeri found poor reproducibility of the TEA based on surgeons identifying these landmarks. In short, a navigation system using the TEA is limited by the imperfect ability of surgeons to locate the epicondyles. Therefore a more reproducible reference for setting rotation is needed. We compared the reproducibility of digitizing either the TEA or the femoral AP axis to the reproducibility of kinematically determining the navigated knee axis using a modified navigation system. We found that the kinematic method can be significantly more reproducible than the other methods if a neutral, distraction, or external rotation load is applied during knee flexion and extension. However, placing varus, valgus, or internal rotation loads on the leg while running the knee through its arc of motion eliminates the benefit of using the NKA. The similarity of the 95\% confidence intervals for the distraction load ([1.96, $2.47])$ and neutral loading $([2.14,2.71])$ suggests that placing a distraction force on the leg may be required to keep the variability of the NKA low.

To give the surgeons their best chance of digitizing the epicondyles successfully, we used a standard medial parapatellar approach for the open knee surgical condition. While this does not reflect the current trend towards minimally invasive surgery, it strengthens our data as we did not obscure the surgeons' views of anatomic landmarks during the digitization procedure [13]. Digitizing the epicondyles may be even more variable when using minimally-invasive approaches.

We have studied the reproducibility of a new reference axis in the distal femur generated by the Stryker knee navigation system which we termed the navigated knee axis. It remains to be determined what its relationship is to the true TEA and AP axes. Understanding the relationship between the NKA and these landmarks would be critical in determining how to use the navigated knee axis for making decisions about femoral cuts. While we found the average, neutralloading, navigated knee axis in the closed surgical condition across specimens to be $0.04^{\circ}$ internally rotated to the average TEA, this has limited utility given the highly variable TEA measurement. In future studies, CT determination of distal femoral landmarks would be useful in studying this relationship.

One caveat is that while the majority of specimens had significant disease and some deformity, we did not include specimens with large deformities. Would the navigated knee axis be as repeatable in limbs with deformity greater than $10^{\circ}$ ? It is unknown whether the navigated knee axis would deviate further from the TEA in more deformed knees. We specifically selected against some types of deformity with our exclusion criteria in order to develop the technique first. Subsequent studies using CT on more deformed knees would determine the relationship between the true TEA and the navigated knee axis. This would confirm whether or not we were replicating any of the deformity in the reference we use. Using a distraction force, which our data supports, mitigates the possibility that kinematics are determined by the diseased articular surfaces. However, it will be important to follow this up with specimens with more significant deformity to prove or disprove this point.

An additional caveat is that we do not know what effect active muscle forces would have on the determination of the navigated knee axis. As this was a cadaver study, we used passive kinematics with no active muscle contribution to determine the navigated knee axis. This is consistent with the lack of active muscle contraction during knee replacement surgery. The absence of muscle activity, however, may limit the use of this study in evaluating the navigated knee axis as a tool in understanding knee kinematics in living individuals. Also, we do not know what effect the use of a tourniquet would have had on our measurements. Future studies should illustrate the impact of a tourniquet on the kinematic axis determinations.

\section{CONCLUSION}

Kinematically determining the navigated knee axis can be more reproducible than digitizing either the TEA or the femoral AP axis using knee navigation. While varus, valgus, and internal rotation loadings increase the variability of the NKA axis orientation, applying a distraction force to the foot during knee motion maintains the low variability. We hope that defining a reference axis with greater reproducibility than the digitized TEA or digitized femoral AP axis may lead to the development of another tool in reducing error in knee component placement. Because we have not demonstrated the relationship between the navigated knee axis and conventional landmarks and references, more study is clearly needed before introducing this technique clinically.

\section{ACKNOWLEDGEMENTS}

The authors would like to acknowledge Charles Roehm, Chris Gatti, Kristi Overgaard, and Amy Mell of the Department of Orthopaedic Surgery and Dean Mueller of the Anatomical Donations Program at The University of Michigan as well as Harald Boschert, Peter Zimmermann, and José-Luis Moctezuma of Stryker Navigation in Freiburg, Germany, for 
all of their help in this project. Additionally we would like to acknowledge our funding source: Stryker Corporation.

\section{REFERENCES}

[1] J.R. Moreland, "Mechanisms of failure in total knee arthroplasty", Clin. Orthop., vol. 226, pp. 49-64, 1988.

[2] M.A. Ritter, P.M. Faris, E.M. Keating, J.B. Meding, "Postoperative alignment of total knee replacement: its effect on survival", Clin. Orthop., vol. 299, pp. 153-156, 1994.

[3] D.L. Churchill, S.J. Incavo, C.C. Johnson, B.D. Beynnon, "The transepicondylar axis approximates the optimal flexion axis of the knee", Clin. Orthop., vol. 356, pp. 111-118, 1998.

[4] R.A. Berger, H.E. Rubash, M.J. Seel, W.H. Thompson, L.S. Crossett, "Determining the rotational alignment of the femoral component in total knee arthroplasty using the epicondylar axis", Clin. Orthop., vol. 286, pp. 40-47, 1993.

[5] S.K. Chauhan, G.W. Clark, S. Lloyd, R.G. Scott, W. Breidahl, J.M. Sikorski, "Computer-assisted total knee replacement: a controlled cadaver study using a multi-parameter quantitative CT assessment of alignment (The Perth CT Protocol)," J. Bone Joint Surg., vol. 86B, pp. 818-823, 2004.

[6] G. Keene, D. Simpson, Y. Kalairajah, "Limb alignment in computer-assisted minimally-invasive unicompartmental knee replacement", J. Bone Joint Surg., vol. 88B: pp. 44-48, 2004.
[7] R. Hart, M. Janecek, A. Chaker, P. Bucek, "Total knee arthroplasty implanted with and without kinematic navigation", Int. Orthop., vol. 27, pp. 366-369, 2003.

[8] J.Y. Jenny, U. Clemens, S. Kohler, H. Kiefer, W. Konermann, R.K Miehlke, "Consistency of implantation of a total knee arthroplasty with a non-image-based navigation system: a case-control study of 235 cases compared with 235 conventionally implanted prostheses", J. Arthroplasty, vol. 20, pp. 832-839, 2005.

[9] S.K. Chauhan, R.G. Scott, W. Breidahl, R.J. Beaver, "Computerassisted knee arthroplasty versus a conventional jig-based technique: a randomized, prospective trial", J. Bone Joint Surg., vol. 86B, pp. 372-377, 2004.

[10] J.Y. Jenny, C. Boeri, "Low reproducibility of the intra-operative measurement of the transepicondylar axis during total knee replacement", Acta Orthop. Scand., vol.75, pp. 74-77, 2004.

[11] M.A. Katz, T.D. Beck, J.S. Silber, R.M. Seldes, P.A. Lotke, "Determining femoral rotational alignment in total knee arthroplasty: reliability of techniques", J. Arthroplasty, vol. 16, pp. 301-305, 2001.

[12] C.W. Spoor, F.E. Veldpaus, "Rigid body motion calculated from spatial co-ordinates of markers", J. Biomech., vol. 13, pp. 391-393

[13] H. Dolezal, Living in a World Transformed: Perceptual and Performatory Adaptation to Visual Distortion. New York: Academic Press, 1982, pp. 61-73.

(C) Doro et al.; Licensee Bentham Open.

This is an open access article licensed under the terms of the Creative Commons Attribution Non-Commercial License (http://creativecommons.org/licenses/by-nc/3.0/) which permits unrestricted, non-commercial use, distribution and reproduction in any medium, provided the work is properly cited. 